\title{
Spin Rotation by Resonant Electric Field in Few-Level Quantum Dots: Floquet Dynamics and Tunneling
}

\author{
D.V. Khomitsky, ${ }^{1, *}$ E.A. Lavrukhina, ${ }^{1}$ and E.Ya. Sherman $\odot^{2,3}$ \\ ${ }^{1}$ Department of Physics, National Research Lobachevsky State University of Nizhni Novgorod, Gagarin \\ Avenue 23, Nizhny Novgorod 603950, Russian Federation \\ ${ }^{2}$ Department of Physical Chemistry, The University of the Basque Country, 48080 Bilbao, Spain \\ ${ }^{3}$ IKERBASQUE Basque Foundation for Science, Bilbao, Spain
}

(Received 2 January 2020; revised 13 June 2020; accepted 15 June 2020; published 29 July 2020)

\begin{abstract}
We study electric dipole spin resonance caused by subterahertz radiation in a multilevel finite-size quantum dot formed in a nanowire, focusing on the range of driving electric field amplitudes where a strong interplay between the Rabi spin oscillations and tunneling from the dot to continuum states can occur. A strong effect of the tunneling on the spin evolution in this regime occurs due to the formation of mixed spin states. As a result, the tunneling strongly limits possible spin manipulation times. We demonstrate a backaction of the spin dynamics on the tunneling and position of the electron. The analysis of the efficiency of the spin manipulation in terms of the system energy shows that tunneling decreases this efficiency. Fourier spectra of the time-dependent expectation value of the electron position show a strong effect of the spin-orbit coupling on their low-frequency components. This result can be applied to operational properties of spin-based nanodevices and can extend the range of possible spin resonance frequencies to the subterahertz domain.
\end{abstract}

DOI: 10.1103/PhysRevApplied.14.014090

\section{INTRODUCTION}

Implementation of electron spins in nanoscale semiconductors, such as quantum dots (QD) and other heterostructures as scalable hardware elements for quantum information processing [1,2], requires tools for fast and efficient spin manipulation in these systems. A preferable way for such manipulations is related to application by electric rather than magnetic fields since a well-controllable electric field can be produced on demand by modern nanoscale electrical engineering tools. The venue for such a manipulation is offered by either the natural or engineered presence of spin-orbit coupling (SOC) in these semiconductor structures, which leads to the electric dipole spin resonance (EDSR) [3-6], and makes it an efficient tool for the manipulation of electron spins in solids. This ability to manipulate spin by an electric field appears, in general, due to the fact that spin precession is related to the electron displacement caused by the application of an external electric field [7].

There are three basic systems where the electric dipole spin resonance can be observed [8]. First, systems with itinerant electrons in bulk crystals [3-6], surfaces [9] with spin-orbit coupling [10], and artificial semiconductor

\footnotetext{
*khomitsky@phys.unn.ru
}

structures, such as heterojunctions and quantum wells [11] and superlattices [12]. Second, theoretically analyzed in Refs. [13-23] and experimentally realized for electrons [24] and holes [25], systems with carriers in single and double semiconductor quantum dots (a similar approach can be applied for carriers in carbon nanotubes [26]). Because of a strong confinement by the external potential, here the spectrum of carriers is discrete, and the carrier always remains in the dot, either two [24] or one dimensional [27], even when a strong electric field is applied. Third, systems with potentials vanishing at large distances, e.g., electrons (holes) on donors (acceptors) [28-31], in finite-range potential produced by various charged gates [32-36], and in surface-based self-assembled quantum dots [37-39].

An important example of spin manipulation in systems with the confining potential vanishing at large distances is given by gate-defined quantum dots [40] and $\mathrm{P}$ donors in Si [41], where the hyperfine coupling to donor nuclear spins results in experimentally achieved possibilities of spin qubit manipulations [42-46]. In a spin ensemble of these donors, the coherent Rabi dynamics have been observed [47]. However, the effect of SOC in these systems is weaker than that achievable in other nanostructures, such as InSb quantum wires and dots and other materials with large tunable SOC. Since the large SOC generally leads to a faster spin flip and shorter operating times, these 
semiconductor structures are of interest for applications, and will be considered here in detail.

This third type of system is of interest since lowfrequency electric fields can ionize the electron states by tunneling and, thus, cause additional nontrivial spin dynamics resulting from the spatial spread of the electron wave function. For example, even for the interminima tunneling in a double quantum dot at relatively short distances, the coupled spin and spatial dynamics may cause strong nonlinearities in the spin-precession Rabi frequency dependence on the driving field amplitude [16].

We are therefore interested in Floquet dynamics in a tunneling system, where a combination of two long time scales becomes important. The first long time scale is the time of spin flip driven by the electric field, inversely proportional to the field amplitude. The second long time scale is the tunneling time due to driving by the electric field, producing coupling of localized states to the continuum, being strongly, approximately exponentially, dependent on the inverse field amplitude. If both these times are of the same order of magnitude, the coupled spin and charge density dynamics studied in this paper become highly nontrivial. In a multilevel quantum dot the tunneling is a well-defined semiclassical process, in contrast to that in shallow quantum dots [48]. As a result of a stronger confinement, the amplitude of the electric field required to operate a spin flip is considerably larger and, therefore, the involvement of the continuum states is qualitatively different.

A relevant issue here is the range of frequencies required for the spin resonance. The major part of experimental setups produces frequencies within the tens of gigahertz range. However, fast and decoherence-robust spin manipulations may require higher subterahertz frequencies. This is because the possible spin Rabi frequencies decrease with decreasing Zeeman splitting and, therefore, will require stronger driving fields. A combination of progress in the development of terahertz sources [49-51] and the demand for high driving frequencies for the EDSR in two-dimensional systems [52] and for fast spin manipulation in conventional nanostructures make these techniques applicable in spin-manipulation technologies.

An interesting aspect of these dynamics is that usually the EDSR assumes a pure spin state, defined on the Bloch sphere, where the spin rotation occurs with a minor change in the electron orbital state. However, in the presence of spin-orbit coupling the spin states become mixed. This circumstance strongly modifies the entire spin evolution. For applications, the efficiency of the spin-flip process, that is, the ratio of the Zeeman energy to the total energy acquired by the electron as a result of the electric field action, is of interest. We demonstrate how the efficiency depends on the system parameters. Moreover, the spinorbit coupling causes a feedback of the spin dynamics on the tunneling efficiency and resulting electron position.
Even at a relatively weak spin-orbit coupling this effect can be strong, as we demonstrate below.

This paper is organized as follows. In Sec. II we introduce the Hamiltonian of our system and discuss timeindependent and periodic contributions to the electron energy. In Sec. III we describe the computational model of electron states and their dynamics and provide the specific system parameters. In Sec. IV the main results are presented: spin dynamics, structure of the electron wave function, time dependencies of the mean energy and efficiency of the spin flip, localization probabilities, and coordinate evolution and its Fourier power spectra. Finally, in Sec. V we give our conclusions and discuss device designs that use the effects described in this paper.

\section{HAMILTONIAN AND OBSERVABLES}

We consider a narrow nanowire, elongated along the $x$ axis located on the top of a doped substrate, as schematically presented in Fig. 1. Assuming that the transversal modes are not excited, we characterize the electron motion by a two-component wave function $\psi(x, t)=$ $\left[\psi_{1}(x, t), \psi_{2}(x, t)\right]^{\top}$, where 'T, denotes transposition, and use the effective mass approximation Hamiltonian with

$$
H(t)=\frac{\hbar^{2} k^{2}}{2 m}+V(x, t)+\frac{\Delta}{2} \sigma_{z}+\alpha \sigma_{y} k .
$$

Here $m$ is the electron effective mass and $k=-i \partial / \partial x$ is the wave vector operator. The time-dependent potential is the sum of the static confinement potential $U(x)$, as can be produced by the electrostatic gate shown schematically by a rectangle above the nanowire in Fig. 1, and the external driving, giving

$$
V(x, t)=U(x)+F x \sin \omega_{d} t,
$$

where $F \equiv e \mathcal{E}_{0}$ with $e$ the fundamental charge and $\mathcal{E}_{0}$ the field amplitude. The Rashba SOC $H_{R}=\alpha \sigma_{y} k$, while more complicated forms of spin-orbit coupling can be found in Refs. [53-56]. The constant magnetic field $\mathbf{B}=(0,0, B)$ produces the Zeeman term in the Hamiltonian. Here the Zeeman splitting $|\Delta|=\mu_{B}|g B|$, where $\mu_{B}$ is the Bohr

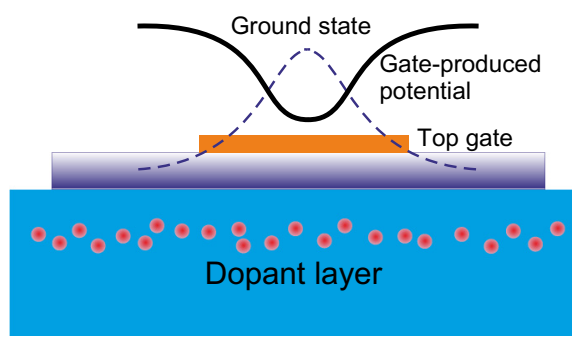

FIG. 1. Schematic presentation of a structure including a gated nanowire on a doped bulk substrate. 
(a)

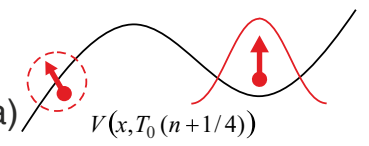

(b)

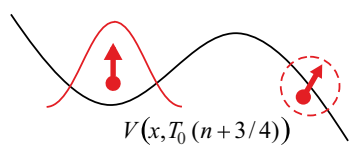

FIG. 2. Schematic illustration of combined tunneling and spin precession caused by a periodic external field. Arrows in the circles located at the points of tunneling-induced escape show that, dependent on the direction of the tunneling, spin precesses in opposite directions.

magneton and $g$ is the Landé factor. The spin-split Zeeman partners participate in the spin resonance driven by the external periodic electric field in Eq. (2). The reason for this participation is that, owing to the presence of SOC, the eigenstates of Eq. (1) at $F=0$ always contain both spin components. Therefore, spin-flip transitions can still be caused by the electric field described with the position operator, producing the EDSR. In this case, the well-defined spin Rabi oscillations occur if one neglects the tunneling effects, that is, coupling to the other orbital and continuum states. In the following sections we discuss the nontrivial effects of the dynamical interaction between discrete and continuum states during the evolution driven by the periodic electric field. One of the effects is illustrated schematically in Fig. 2: in the presence of SOC the direction of spin precession is different, depending on the direction of the tunneling escape, making a controlled spin flip a rather challenging task, especially if the tunneling is triggered by the alternating field responsible for the spin evolution.

To describe the evolution, we solve the nonstationary Schrödinger equation

$$
i \hbar \partial_{t} \psi(x, t)=H(t) \psi(x, t),
$$

where $\partial_{t} \equiv \partial / \partial t$, and calculate expectation values for experimentally measurable observables $\mathcal{O}$ as

$$
\langle\mathcal{O}(t)\rangle=\int_{-\infty}^{\infty} \boldsymbol{\psi}^{\dagger}(x, t) \mathcal{O} \psi(x, t) d x
$$

The observables will be specified later in the text.

\section{MODEL AND NUMERICAL APPROACH}

\section{A. Basis states and model of the dynamics}

We choose the static potential $U(x)=-U_{0} / \cosh ^{2}(x / d)$, assuming that it is deep such that the parameter $\xi \equiv$ $U_{0} m d^{2} / \hbar^{2} \gg 1$. Although the exact bound states for this potential are well known analytically [60] in terms of the Legendre functions, it is practical to consider simple approximations based on the expansion at $x \ll d$ as $U(x)=-U_{0}\left(1-x^{2} / d^{2}\right)$. As a result, the lowest bound states can be described in terms of a harmonic oscillator with the energy interval $\hbar \omega_{\mathrm{HO}}=U_{0} / \sqrt{\xi / 2} \ll U_{0}$ and the Gaussian width $d /(2 \xi)^{1 / 4} \ll d$. The numerically accurate eigenenergies $E_{n}^{(0)}$ and basis states of the Hamiltonian $\hbar^{2} k^{2} / 2 m+U(x)$ are found by expansion in the basis of the periodic functions $\sin \left(x / \lambda_{n}+\delta_{n}\right)$, where $\lambda_{n}=2 L /(\pi n)$ and $\delta_{n}=\pi n / 2, n=1,2, \ldots$, satisfying zero boundary conditions at $x= \pm L$, where $2 L$ is a large wire length.

We then numerically produce the basis of twocomponent eigenstates $\phi_{n}(x)$ of the spinful Hamiltonian

$$
H_{0}=\frac{\hbar^{2} k^{2}}{2 m}+U(x)+\frac{\Delta}{2} \sigma_{z}+\alpha \sigma_{y} k
$$

with spin-split eigenenergies $E_{n}$ such that $H_{0} \phi_{n}(x)=$ $E_{n} \phi_{n}(x)$. As a result, the evolution of the wave function is

$$
\psi(x, t)=\sum_{n} c_{n}(t) e^{-i E_{n} t / \hbar} \phi_{n}(x) .
$$

Thus, the problem is reduced to obtaining a full set of coefficients $c_{n}(t)$ by numerical solution of a large system of linear differential equations, which we present below using some computational details from Ref. [48]. Here we choose the interval $2 L=320 d$ and take 4000 basis states $\phi_{n}(x)$. The initial state is chosen as $\psi(x, 0)=\phi_{1}(x)$, that is, the ground state of $H_{0}$, where, for the realistic parameters, we have $\left\langle\sigma_{z}(0)\right\rangle \approx 1$.

\section{B. System parameters}

In the following analysis we consider a gate-formed quantum dot based on the InSb quantum wire; see Fig. 1. For this material, $m=0.0136$ of a free electron mass [57] and $g=-50.6$. The magnitude of SOC $\alpha$ in InSb can be tuned by the gate voltage up to $100 \mathrm{meVnm}[58,59]$.

For the quantum dot parameters, we accept $U_{0}=27$ $\mathrm{meV}$ and $d=50 \mathrm{~nm}$ with five discrete quantum dot levels $E_{1}^{(0)}, \ldots, E_{5}^{(0)}$ being formed [60] with $\omega_{0}=\left(E_{2}^{(0)}-\right.$ $\left.E_{1}^{(0)}\right) / \hbar=13.43 \mathrm{ps}^{-1}$, and the associated period $T_{0}=$ $2 \pi / \omega_{0}=0.468 \mathrm{ps}$ gives the natural time scale for the evolution. We consider the magnetic field $B=0.447 \mathrm{~T}$, providing the Zeeman splitting of the ground state corresponding to the resonant driving frequency (including the contribution of spin-orbit coupling) $\omega_{d}=2 \mathrm{ps}^{-1}(0.32 \mathrm{THz})$, giving the ratio to the fundamental frequency $\omega_{d} / \omega_{0}=$ 0.149 . Such Zeeman splitting requires a subterahertz-range driving hardware, as discussed in the Introduction. The advantages of this large splitting arise from the following circumstances. It is known that smaller Zeeman splitting gives smaller values of spin-flip matrix elements of the nonstationary part in Eq. (2) [48], which results in a higher 
driving field amplitude $F$ required to operate the spin flip in a desired time. As a result, the leakage to the continuum will be strongly enhanced, as we show below. Thus, the larger driving frequencies may provide the desired spin-flip time with lower operating field, which fosters the applicability of the proposed mechanism.

We consider the effect of a relatively weak SOC $\alpha=$ $5 \mathrm{meVnm}$ and a relatively strong $\alpha=25 \mathrm{meVnm}$ for various driving amplitudes. Taking into account the material parameters for spin-obit coupling produced by an external electric field in InSb [61], we find that the effective twodimensional donor dopant layer concentration, as shown in Fig. 1, corresponding to this spin-orbit coupling is in the range of $10^{11}-10^{12} \mathrm{~cm}^{-2}$. Since the nonuniform static electric field forming the quantum dot has a $z$ component, it can contribute to the Rashba coupling. Taking into account the fact that this field component cannot exceed approximately $U_{0} / e d$, and using the corresponding material parameter [61], we find that this contribution to the SOC is less than $2.5 \mathrm{meVnm}$, considerably smaller than the accepted values for the InSb nanowire.

Now we discuss the effect of the Dresselhaus SOC, typical for III-V semiconductors. The corresponding Hamiltonian has the form $H_{D}=\alpha_{D}(\kappa \sigma)$, where $\alpha_{D}$ is the coupling constant and $\kappa_{x}=k_{x}\left(k_{y}^{2}-k_{z}^{2}\right)$, with other components obtained by cyclic permutation. This $\kappa$-dependence makes the Dresselhaus SOC in nanowires strongly dependent on their shape and orientation. For example, for the wire grown along the crystallographic $x$ axis, we obtain $H_{D}^{[x]}=\alpha_{D} k_{x}\left(\left\langle k_{y}^{2}\right\rangle-\left\langle k_{z}^{2}\right\rangle\right)$, where $\left\langle k_{i}^{2}\right\rangle$ is the shapedependent expectation value of the corresponding operator $[62,63]$. As a result, this coupling can be made zero for the highly symmetric wires of the appropriate cross section. For this growth direction, the Dresselhaus coupling is similar to the Rashba coupling in Eq. (5). For the wires grown along the $y$ or $z$ axis, it will modify only the direction of the SOC field without considerable changes to the spin dynamics. Taking into account the estimate $\alpha_{D} \approx 0.76 \mathrm{eVnm}^{3}$ [61] we obtain, for typical values $\left(\left\langle k_{y}^{2}\right\rangle-\left\langle k_{z}^{2}\right\rangle\right) \sim 10^{-2} \mathrm{~nm}^{-2}$, a coupling of the order of 10 meVnm, not considerably modifying the parameters of the driving fields of interest for the present work.

\section{SPIN DYNAMICS AND FEEDBACK ON THE TUNNELING AND POSITION}

\section{A. Time dependence of the spin: the role of the tunneling}

In Fig. 3 we show the time dependence of $\left\langle\sigma_{z}(t)\right\rangle$ corresponding to Eq. (4) with $\mathcal{O}=\sigma_{z}$, giving

$$
\left\langle\sigma_{z}(t)\right\rangle=\sum_{n_{1}, n_{2}} c_{n_{1}}^{*}(t) c_{n_{2}}(t) e^{-i\left(E_{n_{2}}-E_{n_{1}}\right) t / \hbar}\left\langle\phi_{n_{1}}\left|\sigma_{z}\right| \phi_{n_{2}}\right\rangle
$$
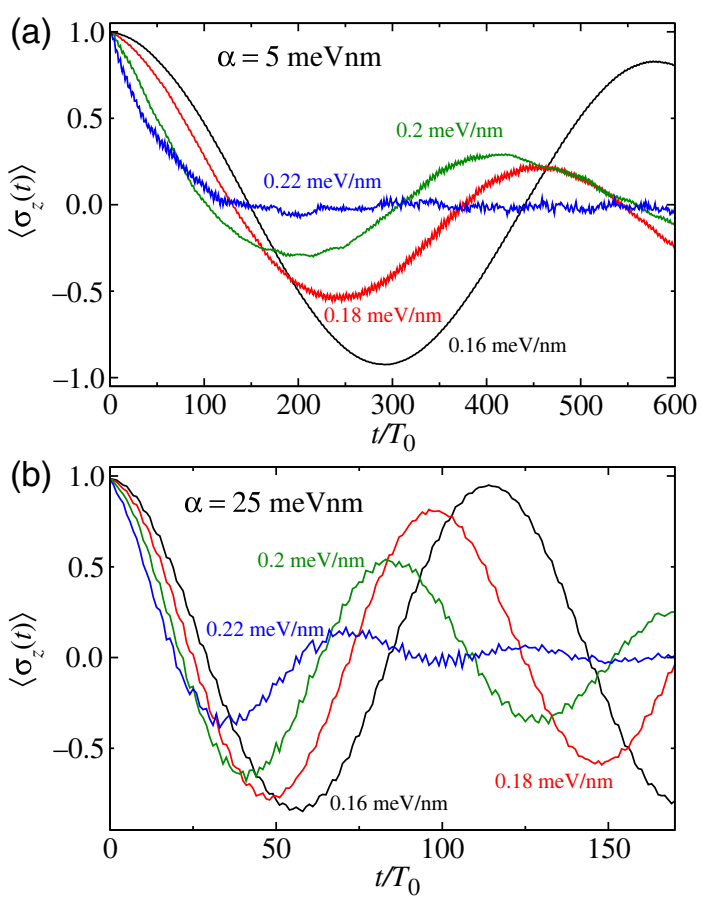

FIG. 3. Spin projection $\left\langle\sigma_{z}(t)\right\rangle$ for $\alpha=5 \mathrm{meVnm}$ (a) and $\alpha=$ $25 \mathrm{meVnm}$ (b) for various driving field amplitudes as marked near the curves. At a relatively high $F \geq 0.2 \mathrm{meV} / \mathrm{nm}$ the spin dynamics demonstrate visible damping of the Rabi oscillations, as caused by the interaction with continuum states increasing with the driving field amplitude.

Here and below the time is measured in units of $T_{0}$, and we track the evolution on calculation time scales of about $650 T_{0}$ for small SOC and $200 T_{0}$ for large SOC. These ranges are determined by the spin-flip process, which needs to be well developed. As a result, we can obtain from the spin dynamics the characteristic spin evolution time. We can see that a sizable decrease in this time can be observed for a moderate increase in $F$ from 0.16 to $0.2 \mathrm{meV} / \mathrm{nm}$, which is in the framework of usual Rabi frequency dependence on the driving field amplitude. When the field grows further, the electron escapes the quantum dot quickly, before well-established Rabi spin oscillations are developed. This effect is stronger at lower values of SOC $\alpha=5 \mathrm{meVnm}$. We can see also that at fields $F \geq$ $0.2 \mathrm{meV} / \mathrm{nm}$ the Rabi oscillations are accompanied by visible damping. We attribute this effect to the interactions between localized and continuum states, which is enhanced at higher driving fields. The continuum states form a dense set of levels with alternating spin projections due to the Zeeman splitting [48]. Transitions to these states driven by an external electric field lead to the wave function consisting of spin-up and spin-down states, and their relative contributions to the total norm have a tendency to equalize when the electron is pushed into the continuum. 
To understand the interplay between tunneling and spin flip, we first evaluate the upper limit for the semiclassical tunneling rate $w_{\text {tun }}$ in the model triangular potential $F x$, neglecting tunneling from excited states, as [64]

$$
\ln \frac{\omega_{0}}{w_{\text {tun }}}<\frac{4 \sqrt{2}}{3} \xi^{1 / 2} \frac{U_{0}}{F d}
$$

Equation (8) gives the upper estimate of the quantity since the real tunneling barrier is lower and more narrow than the triangular barrier. Although this equation shows that the tunneling probability rapidly increases at $F d$ being a sizable fraction of $U_{0}$, the actual tunneling rate, very strongly $F$ dependent, can only be obtained numerically. Since the spin-flip Rabi frequency $\Omega_{R}$ is a linear function of $F$ (being strictly linear when the two-level approximation is applicable) with $\Omega_{R} T_{0} \ll 1$, the effect of tunneling on the spin-flip processes occurs in a relatively narrow range of the driving fields when the two rates are comparable.

For a stronger Rashba coupling, the spin dynamics become faster and well-defined Rabi oscillations have enough time to develop before the tunneling occurs, as can be seen in Fig. 3(b). However, when the field exceeds 0.2 $\mathrm{meV} / \mathrm{nm}$, the electron tunnels from the dot so quickly that the spin flip has no time to be established, regardless of the spin-orbit coupling strength, and the continuum states play the dominant role. In other words, high values of $F$ produce the tunneling rate $w_{\text {tun }}$ [see Eq. (8)] satisfying the inequality $\Omega_{R} \lesssim w_{\text {tun }}$, where the spin-flip time is longer than the tunneling escape time. As to the driving field range desired for controlling spin flips, a small amplitude produces slow Rabi oscillations, which on long times may be hampered by various decoherence processes. So, it is one of our goals to accurately estimate the optimal range of electric fields where the driving is strong enough to trigger fast spin flips while not being sufficiently strong to cause the quantum dot ionization.

It is important that the spin states we consider are mixed rather than pure. This can be seen with the $2 \times 2$ spin density matrix

$$
\rho(t)=\int_{-L}^{L} \boldsymbol{\psi}(x, t) \boldsymbol{\psi}^{\dagger}(x, t) d x .
$$

At $t=0$, we have $\left|\psi_{2}(x, 0)\right| \ll\left|\psi_{1}(x, 0)\right|$ with $\left\langle\sigma_{z}(0)\right\rangle \approx$ 1 such that the initial spin state has a high purity with $\operatorname{tr} \rho^{2}(0) \approx 1$. Note that a state is pure if and only if $\psi_{1}(x, t)=c \psi_{2}(x, t)$, where $c$ is a complex constant. With the course of time, two components of $\psi(x, t)$ begin to develop different shapes, producing the spin density matrix in Eq. (9) with $\operatorname{tr} \rho^{2}(t)<1$ characterizing a mixed spin state [65]. In Fig. 4 we show an example of the structure of mixed spin states in the driven spin dynamics at the end of the computational evolution $t=655 T_{0}$ for $\alpha=5$ $\mathrm{meVnm}$ and $F=0.2 \mathrm{meV} / \mathrm{nm}$. Although at this time and

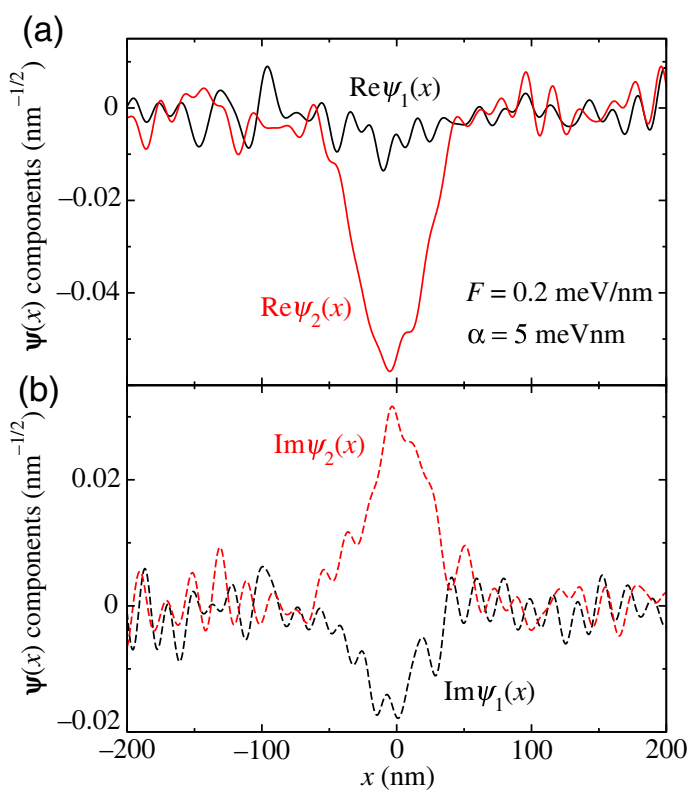

FIG. 4. Components of the electron spinor $\psi(x, t)$ function at $t=655 T_{0}:$ (a) real parts, (b) imaginary parts.

driving field, the electron density still has a well-defined peak inside the confining potential, a broad contribution of the continuum states with a relatively low total probability density has already been formed at $d<|x|<L$, possessing more than half of the total norm.

It can be seen from Fig. 4 that $\psi_{1}(x, t)$ and $\psi_{2}(x, t)$ are strongly different, in both the real and imaginary parts. The densities of the spin components $\left|\psi_{1}(x, t)\right|^{2}$ and $\left|\psi_{2}(x, t)\right|^{2}$ (not shown in the figure) are shifted with respect to each other, corresponding to Fig. 2, where the direction of the tunneling determines the direction of the spin precession.

By evaluating the spin-flip times in Fig. 3 we note that they are of the order of $(30 \ldots 40) T_{d}$ for $\alpha=5 \mathrm{meVnm}$ and $(5 \ldots 8) T_{d}$ for $\alpha=25 \mathrm{meVnm}$, where $T_{d}=6.71 T_{0}$ is the driving field period. A difference to Ref. [48] is that the electric field amplitude required to operate a spin flip in a multilevel dot is three orders of magnitude larger than that for the shallow dot. We attribute these differences to the considerably stronger localization and energy scales of a multilevel dot, where one needs larger electric fields to induce the spin flip in a desired time. These larger fields lead to a broader energy interval of the continuum states contributing to the position and spin evolution.

To summarize this subsection, we mention that, with the expression for spin-flip matrix elements of coordinate $[28,29]$ for the Zeeman-split ground state in quantum dots of interest, one can estimate the tunneling-limited maximal Rabi spin frequency $\Omega_{R}^{[\max ]}$ as $\Omega_{R}^{[\max ]} \sim \omega_{d} d / L_{\mathrm{SO}}$, where $L_{\mathrm{SO}}=\hbar^{2} / m \alpha$ is the spin-precession length. In general, the length $L_{\mathrm{SO}}$ is determined by the joint effect of the Rashba 
and Dresselhaus couplings, and the existence of this maximal Rabi frequency is model independent. Taking into account the fact that, for our set of parameters at $\alpha=5$ meVnm, $L_{\mathrm{SO}} \approx 10^{-4} \mathrm{~cm}$, we obtain good agreement with the numerical results presented in Fig. 3. Note that such a limit has to exist for all finite-potential systems, including $\mathrm{P}$ donors in Si. However, every realization needs a special analysis, which can be a topic of a further research.

\section{B. Efficiency of the spin flip}

The studies of coupled spin and coordinate dynamics can be facilitated by tracking the time dependence of the energy-related variables, such as $\langle E(t)\rangle$ defined as

$$
\langle E(t)\rangle=\sum_{n}\left|c_{n}(t)\right|^{2} E_{n},
$$

where the sum is taken over all basis states with coefficients $c_{n}(t)$ in the wave function (6) for $E_{n}$ defined with Eq. (4) for the unperturbed Hamiltonian (5).

With the external driving, the energy is pumped into the system, and the period-averaged expectation value of $\langle E(t)\rangle$ grows with time. When $\langle E(t)\rangle$ passes the threshold $\langle E(t)\rangle=0$ between the localized and continuum states, the electron has effectively tunneled from the quantum dot into the continuum.

In Fig. 5(a) we plot $\langle E(t)\rangle$ for $\alpha=5 \mathrm{meVnm}$ and the same electric fields as in Fig. 3, and in Fig. 5(b) we show examples for fixed $F=0.2 \mathrm{meV} / \mathrm{nm}$ and different SOC parameters. As expected, the tunneling time quickly decreases with increasing field amplitude, and for strong fields greater than $0.2 \mathrm{meV} / \mathrm{nm}$, the electron escapes into the continuum during approximately ten periods of driving. The influence of SOC on the energy evolution can be illustrated by Fig. 5(b): at the given time interval and at zero SOC the energy grows faster than for finite SOC presented in the figure. This effect can be attributed to stronger coupling of all spin-resolved $\alpha$-dependent states via SOC, which slows to some extent the motion to higher energy states.

In addition to the time dependence $\langle E(t)\rangle$, it is of interest to study the spin-flip efficiency $\eta(t)$ defined as the ratio of the energy pumped into the spin degree of freedom to the total change of the mean energy:

$$
\eta(t)=\frac{\Delta}{2} \frac{\left\langle\sigma_{z}(t)\right\rangle-\left\langle\sigma_{z}(0)\right\rangle}{\langle E(t)\rangle-\langle E(0)\rangle} .
$$

Here $\langle E(0)\rangle \equiv E_{1}$. In Fig. 6 we show this $\eta(t)$ for some of the electric fields from Fig. 3. We can see that its time dependence is qualitatively independent of the SOC strength. It reaches the maximum when the first full or almost full spin flip is achieved. After this, the magnitude of $\eta(t)$ decreases with time. The explanation is straightforward: the spin dynamics and in particular the
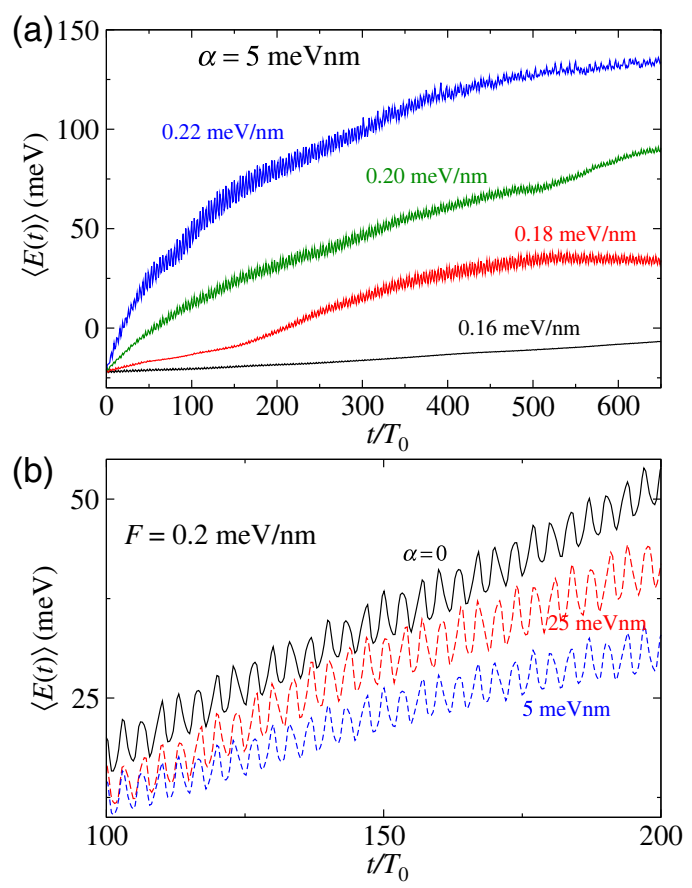

FIG. 5. Time dependence of the mean energy $\langle E(t)\rangle$ for (a) SOC parameter $\alpha=5 \mathrm{meVnm}$ and the same electric field amplitudes as in Fig. 3, and (b) fixed $F_{0}=0.2 \mathrm{meV} / \mathrm{nm}$ and different SOC parameters $\alpha=0, \alpha=5$, and $\alpha=25 \mathrm{meVnm}$.

Zeeman energy have an oscillating character (at low driving strength) or oscillating plus decaying character (at high driving strength). So, the numerator in Eq. (11) does not grow with time, while the denominator in general increases, as can be seen from the energy dependence in Fig. 5. As to the magnitude of Eq. (11), it is greater for a larger SOC strength since the stronger SOC provides a quicker spin flip with lower corresponding change in the mean energy, giving the smaller denominator in Eq. (11) at the moment when the maximum numerator is reached.

\section{Feedback on the tunneling}

To visualize the electron escape from the quantum dot, we use the stay probability that defines the weight of the localized eigenfunctions in the discrete part of the spectrum as

$$
P(t)=\sum_{i(\text { loc })}\left|c_{i}(t)\right|^{2}
$$

where the summation is taken over the contribution of localized $\phi_{i}(x)$ states.

The localization probability (12) is shown in Fig. 7 for two time intervals. We can see that, for the short evolution times shown in Fig. 7(a), both values of the SOC strength produce similar evolution $P(t)$, and the values of Eq. (12) for the smaller $\alpha$ are in general slightly higher than for the larger $\alpha$. The situation is, however, somewhat different on 

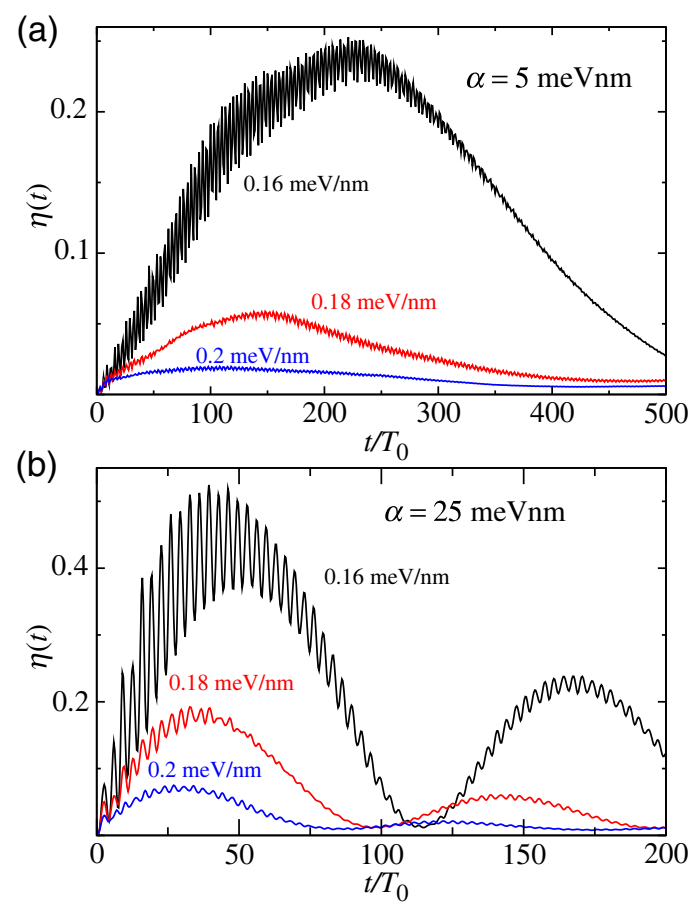

FIG. 6. Time dependent spin-flip efficiency (11): (a) $\alpha=5$ meVnm and (b) $\alpha=25 \mathrm{meVnm}$ for some of the driving fields (marking the plots) from Fig. 3.

long times to $600 T_{0}$ shown in Fig. 7(b) for small $\alpha=5$ meVnm together with the $\alpha=0$ realization. Here two $P(t)$ curves for a given driving strength and different SOC swap more frequently than in Fig. 7(a).

Important elements for understanding the feedback effect of the spin motion on the time-dependent position and tunneling are the anomalous [66] spin-dependent velocity [60]

$$
v_{\mathrm{SO}}=\frac{i}{\hbar}\left[\alpha \sigma_{y} k, x\right]=\frac{\alpha}{\hbar} \sigma_{y}
$$

and the corresponding acceleration

$$
\frac{d}{d t} v_{\mathrm{SO}}=\frac{i}{\hbar} \frac{\Delta}{2} \frac{\alpha}{\hbar}\left[\sigma_{z}, \sigma_{y}\right]=\frac{\alpha \Delta}{\hbar^{2}} \sigma_{x}
$$

This term leads to variation in the velocity due to the spin precession, as was observed in experiments on high-frequency conductivity of two-dimensional electron gas [67], and the effect of time dependent $\left\langle\sigma_{y}(t)\right\rangle$ on $\langle x(t)\rangle$ appears as a result. The corresponding local probability flux satisfying the equation $\partial_{t}\left(\boldsymbol{\psi}^{\dagger}(x, t) \boldsymbol{\psi}(x, t)\right)+$ $\partial_{x} j(x, t)=0$, where $\partial_{x} \equiv \partial / \partial x$, is given by

$$
\begin{aligned}
j(x, t)= & \frac{i \hbar}{2 m}\left[\boldsymbol{\psi}(x, t) \partial_{x} \boldsymbol{\psi}^{\dagger}(x, t)-\boldsymbol{\psi}^{\dagger}(x, t) \partial_{x} \psi(x, t)\right] \\
& +\frac{\alpha}{\hbar} \boldsymbol{\psi}^{\dagger}(x, t) \sigma_{y} \boldsymbol{\psi}(x, t) .
\end{aligned}
$$
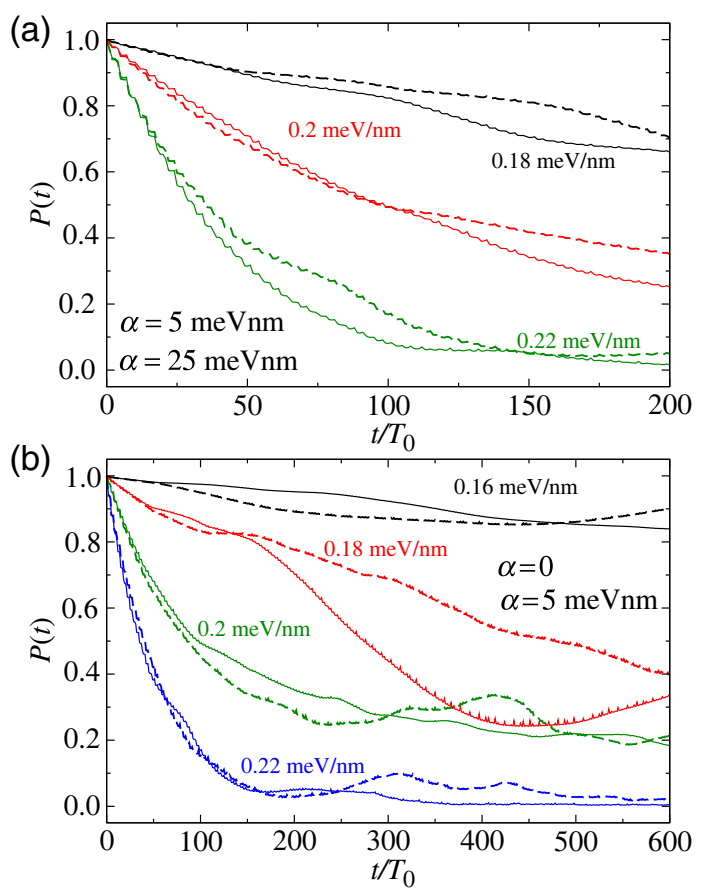

FIG. 7. Time dependence of localization probability (12) for (a) short evolution times with $\alpha=5 \mathrm{meVnm}$ (dashed lines) and $\alpha=25 \mathrm{meVnm}$ (solid lines), and (b) long evolution times with $\alpha=0$ (solid lines) and $\alpha=5 \mathrm{meVnm}$ (dashed lines). The plots are marked with the values of the driving field amplitudes.

Note that, for a relatively small coupling constant $\alpha=$ $5 \mathrm{meVnm}$, the maximum value of the $v_{\text {SO }}$ velocity $\alpha / \hbar \approx$ $8 \mathrm{~nm} / \mathrm{ps}$ can sufficiently modify the tunneling process.

\section{Evolution of the position}

\section{Time dependence of the expectation values}

Along with the spin evolution, the expectation value of the coordinate $\langle x(t)\rangle$ is of interest for understanding the dynamics. It provides information of the electron localization domain and can help in studies of the tunneling effect. For example, the sufficient criteria for tunneling into the continuum may be formulated in terms of the $\langle x(t)\rangle$ amplitude: if it steadily exceeds the quantum dot size $d$, the electron is out of the dot. Also, this expectation value produces a time-dependent electric field outside of the quantum dot, which under certain conditions, can be experimentally tracked.

We calculate the dynamics of $\langle x(t)\rangle$ in analogy with Eq. (7), where the spin operator is replaced by $x$, with some examples of coordinate dynamics presented in Fig. 8. The upper panel shows the dynamics for the low amplitude $F=0.16 \mathrm{meV} / \mathrm{nm}$ and the bottom panel shows the dynamics for $F=0.22 \mathrm{meV} / \mathrm{nm}$. We can conclude from Fig. 8(a) that, for low driving amplitude $F=0.16 \mathrm{meV} / \mathrm{nm}$, the electron is still confined within the quantum dot, and its spin exhibits well-defined Rabi oscillations (see Fig. 3). 

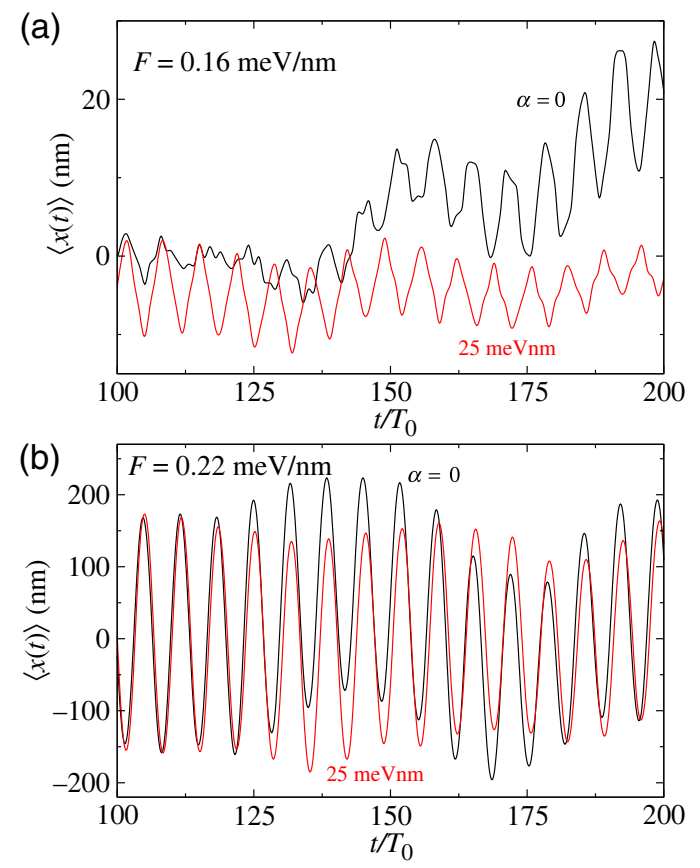

FIG. 8. Time dependence of the mean value of the coordinate $\langle x(t)\rangle$ for $\alpha=0$ and high $\alpha=25 \mathrm{meVnm}$, shown for the driving field amplitude (a) $F=0.16 \mathrm{meV} / \mathrm{nm}$ and (b) $F=$ $0.22 \mathrm{meV} / \mathrm{nm}$. For low driving amplitude $F=0.16 \mathrm{meV} / \mathrm{nm}$, $|\langle x(t)\rangle| \ll d$, while for $F=0.22 \mathrm{meV} / \mathrm{nm}$, the amplitude of $\langle x(t)\rangle$ exceeds the quantum dot size. Here $\max \{|\langle x(t)\rangle|\}<3 d$, meaning that the electron motion is still strongly influenced by the $U(x)$ potential and approximately limited by the tunneling escape points at $\pm E_{1} / F$, where $E_{1}$ is the ground state energy.

When the driving amplitude grows, the delocalization trend is manifested, and for the highest amplitude $F=$ $0.22 \mathrm{meV} / \mathrm{nm}$, the amplitude of $\langle x(t)\rangle$ well exceeds the quantum dot size, as can be seen in Fig. 8(b).

We compare the results presented in Fig. 8 with the evolution of the localization probability (12) in Fig. 7. It is clear that, for the given time $t$, the localization probability is significantly lower at higher driving fields as manifested both in small $P(t)$ and in large $\langle x(t)\rangle$ amplitudes. Thus, the delocalization trend is tracked simultaneously in the coordinate and Hilbert spaces. The strong dependence of the delocalization (ionization in atomic physics) probability on the low-frequency electric field amplitude has an exponential character [64], albeit with a rather undetermined model-dependent prefactor. Thus, our numerical findings shed light on the amplitudes above which an efficient tunneling to the continuum takes place. In addition, we note that despite two possible directions of escape in a periodic field, the probability density becomes broad, but remains single peaked, as can be seen in Fig. 4, making $\langle x(t)\rangle$ a reliable characteristic of the expectation value of the electron position.
The displacements presented in Fig. 8 can be compared with the results for the oscillation amplitude $x_{0}^{[\text {loc }]}$ for a particle localized in the potential $U_{0} x^{2} / d^{2}$, and a free electron driven by a periodic field, $x_{0}^{[\mathrm{cl}]}$, where

$$
x_{0}^{[\mathrm{loc}]}=\frac{d^{2}}{2 U_{0}} F, \quad x_{0}^{[\mathrm{cl}]}=\frac{1}{m \omega_{d}^{2}} F .
$$

For $F=0.16 \mathrm{meV} / \mathrm{nm}$, the estimated amplitude $x_{0}^{[\mathrm{loc}]} \approx$ $8 \mathrm{~nm}$, which is in good agreement with the calculations at $t<100 T_{0}$ (not shown in the figure). Here the velocity amplitude $v_{0}^{[\text {loc }]}=\omega_{d} x_{0}^{[\text {loc }]} \approx 16 \mathrm{~nm} / \mathrm{ps}$, meaning that the anomalous velocity $\alpha / \hbar$ plays an essential role in the electron dynamics. For a delocalized electron, Eq. (16) yields, for $F=0.22 \mathrm{meV} / \mathrm{nm}$, the amplitude $x_{0}^{[\mathrm{cl}]} \approx 720 \mathrm{~nm}$, which is considerably larger than the calculated values. This difference is due to the fact that in the tunneling ionization a broadly distributed probability density rather than a well-defined wavepacket is being formed, resulting in a relatively small amplitude of $\langle x(t)\rangle$. This can be illustrated by the example for the wave function component profile shown in Fig. 4, which can be compared with the localization probability in Fig. 7(b): for $F=0.2 \mathrm{meV} / \mathrm{nm}$, the low value of the localization probability for the final moment of computation $(P \approx 0.2)$ corresponds to Fig. 4 , which describes a wave function with substantial impact of continuum states, demonstrating a widely delocalized profile rather than a well-localized wavepacket. As to the velocity, taking into account the fact that the numerically obtained amplitude $x_{0}^{[\text {tun] }} \approx 150 \mathrm{~nm}$, we find that the corresponding velocity amplitude $v_{0}^{\text {[tun] }} \approx 300 \mathrm{~nm} / \mathrm{ps}$, being comparable to the maximum anomalous velocity at $\alpha=$ $25 \mathrm{meVnm}$, confirming the importance of spin-orbit coupling for the electron displacement. At this point, we can define the corresponding wavevector $k_{0}^{[\text {tun }]} \equiv v_{0}^{[\text {tun] }} m / \hbar$ and the spin-precession rate $\Omega_{0}^{[\mathrm{tun}]}=2 \alpha k_{0}^{[\mathrm{tun}]} / \hbar$. For the given set of system parameters and $\alpha=5 \mathrm{meVnm}$, we obtain, at $F=0.22 \mathrm{meVnm}, \Omega_{0}^{[\mathrm{tun}]} \approx 0.53 \mathrm{ps}^{-1}$. In Fig. 3(a) we can see that, at $F=0.22 \mathrm{meV} / \mathrm{nm}$, the characteristic time of the spin evolution (the time at which the expectation value $\left\langle\sigma_{z}(t)\right\rangle=0$ ) is close to $45 \mathrm{ps}$, considerably larger than $\pi / \Omega_{0}^{[\mathrm{tun}]} \approx 6 \mathrm{ps}$. This difference means that the main effect of the tunneling on the spin evolution is due to the formation of mixed states rather than due to the driven tunneling-induced spin precession.

By analyzing the results in Figs. 3-8, we can see that the tunneling becomes efficient at a few periods of the driving field when the driving amplitude exceeds the values of about $(0.18 \ldots 0.2) \mathrm{meV} / \mathrm{nm}$. At lower fields the tunneling is slow enough to allow for a well-defined spin flip. For fields higher than $0.22 \mathrm{meV} / \mathrm{nm}$, the potential well opens so effectively that the electron escapes into the continuum before the well-established Rabi oscillations are 
developed. So, we need to choose some optimal intermediate driving fields to achieve a good spin flip in a proper time. Besides, the very strong dependence of the tunneling probability on the driving field amplitude requires a welldefined window of the field amplitude in order to achieve the desired spin flip if one is interested in still keeping the electron inside the hosting quantum dot.

As for the $\alpha$-dependence of the tunneling, we can see that the patterns of the position $\langle x(t)\rangle$, the localization $P(t)$, and the energy $\langle E(t)\rangle$ are similar but still quantitatively different depending on the SOC strength. For example, for a stronger SOC, the localization probability is slightly lower at all times and for most of the driving fields, i.e., the tunneling, in general, becomes faster with increasing SOC. We attribute this effect to a more intense EDSR development at a higher SOC. A stronger SOC enlarges the matrix elements for the states with opposite spins, leading not only to a faster spin flip but also to a larger occupation of higher energy levels, speeding up, to some extent, the tunneling.

\section{Fourier analysis of the dynamics}

It is of interest to study the driven evolution in terms of its spectral properties, namely, the Fourier power spectrum applied to a finite sequence of data for $\langle x(t)\rangle$ collected on the evolution interval $T=N T_{0}$ with $N$ points spaced by $T_{0}$. Here it is appropriate to use the discrete version of the Fourier transform, written as

$$
X_{p}=\sum_{n=0}^{N-1}\left\langle x\left(n T_{0}\right)\right\rangle e^{-i n \omega_{p} / \omega_{0}} .
$$

Here $X_{p}$ is the output for the Fourier harmonic at frequency $\omega_{p}=\omega_{0} p / N$. Eq. (17) gives Fourier data for the frequency interval $0, \ldots, \omega_{0} / 2$ with frequency spacing $\omega_{0} / N$, so the index $p$ takes the values $1, \ldots, N / 2$, where it is convenient to express the frequencies in units of $\omega_{d}$. For our numerical simulations, we have the values $N=200, \ldots, 600$, where the discrete spectra obtained from Eq. (17) form a dense quasicontinuous set of states.

Here we may expect the local maxima at both the driving frequency $\omega_{d}$ and in the low-frequency part associated with the slow spin evolution coupled to the coordinate via SOC. In Fig. 9 we show examples of Fourier power spectra to compare the $\alpha=0$ system with the system having moderate SOC for driving with $F=0.16 \mathrm{meV} / \mathrm{nm}$ and $F=0.22 \mathrm{meV} / \mathrm{nm}$. We see that the presence of SOC modifies mainly the low frequency part of $X_{p}$ in approximately the $10^{-2} \mathrm{THz}$ frequency domain. This is natural due to the SOC of the coordinate with the spin that mainly affects lower frequencies associated with the spin evolution that is lower than the driving frequency of the electric field. The peak at $\omega_{p} / \omega_{d}=1$ is present for all SOC parameters and driving field amplitudes and corresponds to the (a)
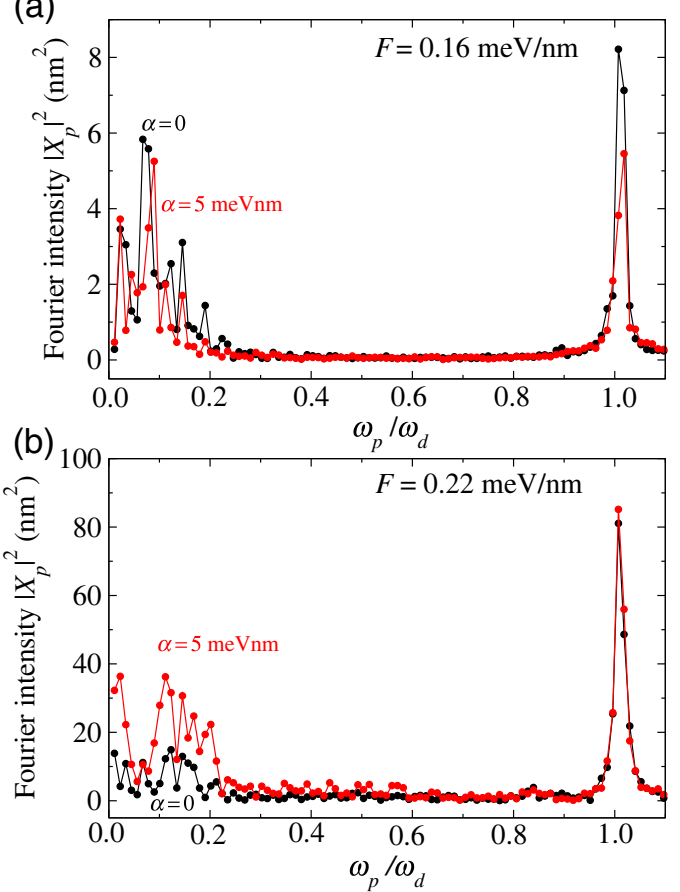

FIG. 9. Fourier intensities $\left|X_{p}\right|^{2}$ for $\alpha=0$ and $\alpha=5 \mathrm{meVnm}$ (as marked near the plots): (a) $F=0.16 \mathrm{meV} / \mathrm{nm}$, (b) $F=0.22$ $\mathrm{meV} / \mathrm{nm}$.

dominant frequency of driven dynamics. Another interesting feature of Fourier spectra is the difference between the zero and nonzero SOC cases that increases at high driving fields, as can be seen by comparing Figs. 9(a) and 9(b). With the increase in the driving field, at $\alpha=0$, the contribution of the large-displacement dynamics at frequency $\omega_{d}$ dominates over the low-frequency part of the displacement spectrum. However, a finite SOC due to the anomalous velocity still produces a relatively intense low-frequency motion.

It is interesting to note that in the nonrelativistic nonlinear atomic optics, frequent recollisions of a driven electron with the singular Coulomb potential of the remaining ion [68] lead to a strong enhancement in the high-frequency radiation. In our calculations we take into account a relativistic effect in the form of the spin-orbit coupling, albeit for a nonsingular potential, where a result of these frequent recollisions is seen as a relatively small amplitude of $\langle x(t)\rangle$ compared to $x_{0}^{[\mathrm{cl}]}$ in Eq. (16), as shown in Fig. 8(b). It will be of future interest to study high-frequency harmonics produced in the presence of these relativistic effects.

\section{CONCLUSIONS AND DISCUSSION}

We study the electric dipole spin resonance with simultaneous tunneling and position evolution in a multilevel quantum dot formed in a nanowire as caused by a driving field in the subterahertz range. We demonstrate a strong 
effect of tunneling on the driven spin-flip processes with disappearance of well-defined Rabi spin oscillations when the tunneling rate is of the order of the corresponding Rabi frequency. For multilevel quantum dots, this matching occurs for electric field amplitudes of the order of 0.1 $\mathrm{meV} / \mathrm{nm}$, being in our realization close to $0.2 \mathrm{meV} / \mathrm{nm}$. Thus, the tunneling induced by the driving electric field strongly limits the maximal spin-flip Rabi frequency and the reliability of the spin manipulation. Although this effect is common for all systems where electrons are localized in a finite-size potential, such as donors, gate-based quantum dots, and lattice defects, every realization requires a system-specific analysis. The strong effect of the tunneling on the spin dynamics is due to the formation of the mixed spin states, shifting the spin vector inside the Bloch sphere. In addition, we demonstrate backaction of the spin dynamics on the tunneling probability and position of the electron. This backaction is qualitatively attributed to the anomalous spin-dependent velocity, proportional to the spin-orbit coupling strength. These effects should be taken into account in the development of techniques for fast operations in the electron spin-based qubit architecture in semiconductor nanostructures.

Finally, we would like to briefly discuss possible device implementation of the above predicted effects. A sketch of a device utilizing the tunneling to the continuum accompanying the spin rotation is shown in Fig. 10. The EDSR spin manipulation for an electron in the QD starts at $t=t_{0}$, and at $t=t_{1}$ the readout begins at the readout area for the electron tunneled to the continuum, as shown in Fig. 10. At the arrival at the readout point, the electron has spin components determined by both the spin manipulation in the dot and the propagation to the readout point. The latter can be accurately found from knowledge of the spin precession in the nanowire, including the spin-precession length discussed in Sec. IV A. Thus, the abilities of spin manipulations can be enhanced by the tunneling process. The readout process shown in Fig. 10 is performed outside of the QD area due to the delocalization of the electron wave function, thus reducing the mutually perturbing effects of

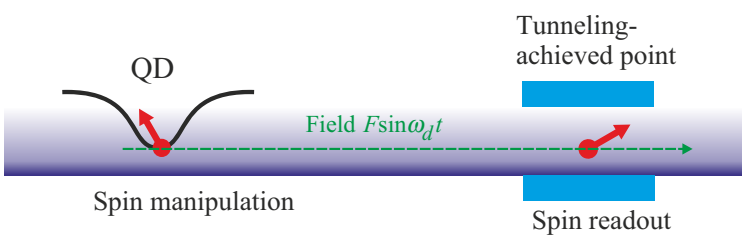

FIG. 10. Sketch of a device utilizing the joint effect of spin rotation and tunneling to the continuum. At $t=t_{0}$ the EDSR spin manipulation starts, and at some $t_{1}>t_{0}$ the readout begins for the electron outside of the dot. The readout process is performed outside of the QD, thus reducing the perturbation effects of the measurement on the electron spin rotation in the dot and, in turn, of the spin rotation on the readout. the EDSR spin manipulation and spin measurement. This is illustrated by Fig. 4, where the sizable out-of-the-dot contribution of the wave function is visible and the corresponding spin components can be detected with high fidelity. Such a setup is different from the setup using the second QD required for spin readout in the EDSR experiments [24,27] where the Pauli spin blockade has been utilized to enhance or suppress the current through the whole structure indicating the spin flip. We expect that our scheme with spatially separated spin rotation and readout intervals can provide alternative ways for experimental spin manipulation setups and their applications.

\section{ACKNOWLEDGMENTS}

D.V.K. and E.A.L. are supported by the Ministry of Science and Higher Education of the Russian Federation through the State Assignment No. 0729-2020-0058. E.A.L. was supported by a grant from the President of the Russian Federation for young scientists under Grant No. MK6679.2018.2. E.Y.S. acknowledges support from the Spanish Ministry of Science, Innovation and Universities, the European Regional Development Fund FEDER through Grant No. PGC2018-101355-B-I00 (MCIU/AEI/FEDER, UE), and the Basque Country Government through Grant No. IT986-16. E.Y.S. is grateful to S. Studenikin for valuable discussions.

[1] D. Loss and D. P. DiVincenzo, Quantum computation with quantum dots, Phys. Rev. A 57, 120 (1998).

[2] G. Burkard, D. Loss, and D. P. DiVincenzo, Coupled quantum dots as quantum gates, Phys. Rev. B 59, 2070 (1999).

[3] E. I. Rashba, Properties of semiconductors with an extremum loop. 1. Cyclotron and combinational resonance in a magnetic field perpendicular to the plane of the loop, Sov. Phys. Solid State 2, 1109 (1960).

[4] G. Bemski, Spin Resonance of Conduction Electrons in InSb, Phys. Rev. Lett. 4, 62 (1960).

[5] R. L. Bell, Electric Dipole Spin Transitions in InSb, Phys. Rev. Lett. 4, 52 (1962).

[6] Y. A. Bratashevskii, A. A. Galkin, and Y. M. Ivanchenko, Resonance absorption by band carriers in InSb, Sov. Phys. Solid State 5, 260 (1963).

[7] T. Čadež, J. H. Jefferson, and A. Ramšak, Exact Nonadiabatic Holonomic Transformations of Spin-Orbit Qubits, Phys. Rev. Lett. 112, 150402 (2014); A. Ramšak, T. Čadež, A. Kregar, and L. Ulčakar, Exact spin-orbit qubit manipulation, Eur. Phys. J. Spec. Top. 227, 353 (2018).

[8] For a review, see: E. I. Rashba and V. I. Sheka, Electricdipole spin resonances, arXiv:1812.01721.

[9] J. Ibañez-Azpiroz, A. Eiguren, E. Ya. Sherman, and A. Bergara, Spin-Flip Transitions Induced by Time-Dependent Electric Fields in Surfaces with Strong Spin-Orbit Interaction, Phys. Rev. Lett. 109, 156401 (2012). 
[10] F. T. Vas'ko, Spin splitting in the spectrum of twodimensional electrons due to the surface potential, JETP Lett. 30, 541 (1980).

[11] E. I. Rashba and Al. L. Efros, Orbital Mechanisms of Electron-Spin Manipulation by an Electric Field, Phys. Rev. Lett. 91, 126405 (2003).

[12] D. V. Khomitsky, Manipulating the spin texture in a spinorbit superlattice by terahertz radiation, Phys. Rev. B 77, 113313 (2008).

[13] V. N. Golovach, M. Borhani, and D. Loss, Electric-dipoleinduced spin resonance in quantum dots, Phys. Rev. B 74, 165319 (2006).

[14] J. H. Jiang, M. Q. Weng, and M. W. Wu, Intense terahertz laser fields on a quantum dot with Rashba spin-orbit coupling, J. Appl. Phys. 100, 063709 (2006).

[15] X. Hu, Yu-Xi Liu, and F. Nori, Strong coupling of a spin qubit to a superconducting stripline cavity, Phys. Rev. B 86, 035314 (2012).

[16] D. V. Khomitsky, L. V. Gulyaev, and E. Ya. Sherman, Spin dynamics in a strongly driven system: Very slow Rabi oscillations, Phys. Rev. B 85, 125312 (2012).

[17] M. Borhani and X. Hu, Spin manipulation and relaxation in spin-orbit qubits, Phys. Rev. B 85, 125132 (2012).

[18] M. P. Nowak, B. Szafran, and F. M. Peeters, Resonant harmonic generation and collective spin rotations in electrically driven quantum dots, Phys. Rev. B 86, 125428 (2012).

[19] Y. Ban, Xi Chen, E. Ya. Sherman, and J. G. Muga, Fast and Robust Spin Manipulation in a Quantum Dot by Electric Fields, Phys. Rev. Lett. 109, 206602 (2012).

[20] Rui Li, J. Q. You, C. P. Sun, and F. Nori, Controlling a Nanowire Spin-Orbit Qubit via Electric-Dipole Spin Resonance, Phys. Rev. Lett. 111, 086805 (2013); Rui Li and J. Q. You, Anisotropic exchange coupling in a nanowire double quantum dot with strong spin-orbit coupling, Phys. Rev. B 90, 035303 (2014).

[21] J. Romhányi, G. Burkard, and A. Pályi, Subharmonic transitions and Bloch-Siegert shift in electrically driven spin resonance, Phys. Rev. B 92, 054422 (2015).

[22] M. T. Veszeli and A. Pályi, Fast electron spin flips via strong subcycle electric excitation, Phys. Rev. B 97, 235433 (2018).

[23] P. Stipsić and M. Milivojević, Control of a spin qubit in a lateral GaAs quantum dot based on symmetry of gating potential, Phys. Rev. B 101, 165302 (2020); S. Miladić, P. Stipsić, E. Dobardžić, and M. Milivojević, Electrical control of a spin qubit in InSb nanowire quantum dots: Strongly suppressed spin relaxation in high magnetic field, Phys. Rev. B 101, 155307 (2020).

[24] K. C. Nowack, F. H. L. Koppens, Yu. V. Nazarov, and L. M. K. Vandersypen, Coherent control of a single electron spin with electric fields, Science 318, 1430 (2007).

[25] S. Studenikin, M. Korkusinski, M. Takahashi, J. Ducatel, A. Padawer-Blatt, A. Bogan, D. Guy Austing, L. Gaudreau, P. Zawadzki, A. Sachrajda, Y. Hirayama, L. Tracy, J. Reno, and T. Hargett, Electrically tunable effective gfactor of a single hole in a lateral GaAs/AlGaAs quantum dot, Commun. Phys. 2, 159 (2019).
[26] E. N. Osika and B. Szafran, Spin-orbit interaction in bent carbon nanotubes: Resonant spin transitions, J. Phys.: Condens. Matter 27, 435301 (2015).

[27] S. Nadj-Perge, S. M. Frolov, E. P. A. M. Bakkers, and L. P. Kouwenhoven, Spin-orbit qubit in a semiconductor nanowire, Nature 468, 1084 (2010).

[28] E. I. Rashba and V. I. Sheka, Combination resonance at local centers of large radius, Sov. Phys. Solid State 6, 114 (1964).

[29] E. I. Rashba and V. I. Sheka, Combination resonance at acceptor centers, Sov. Phys. Solid State 6, 451 (1964).

[30] X. Linpeng, T. Karin, M. V. Durnev, R. Barbour, M. M. Glazov, E. Ya. Sherman, S. P. Watkins, S. Seto, and K.-M. $\mathrm{C}$. $\mathrm{Fu}$, Longitudinal spin relaxation of donor-bound electrons in direct band-gap semiconductors, Phys. Rev. B 94, 125401 (2016).

[31] X. Linpeng, M. L. K. Viitaniemi, A. Vishnuradhan, Y. Kozuka, C. Johnson, M. Kawasaki, and K.-M. C. Fu, Coherence Properties of Shallow Donor Qubits in ZnO, Phys. Rev. Appl. 10, 064061 (2018).

[32] J. H. Davies, I. A. Larkin, and E. V. Sukhorukov, Modeling the patterned two-dimensional electron gas: Electrostatics, J. Appl. Phys. 77, 4504 (1995).

[33] A. G. Malshukov, C. S. Tang, C. S. Chu, and K. A. Chao, Spin-current generation and detection in the presence of an ac gate, Phys. Rev. B 68, 233307 (2003).

[34] L. Serra, D. Sánchez, and R. Lopez, Rashba interaction in quantum wires with in-plane magnetic fields, Phys. Rev. B 72, 235309 (2005).

[35] T. Zibold, P. Vogl, and A. Bertoni, Theory of semiconductor quantum-wire-based single- and two-qubit gates, Phys. Rev. B 76, 195301 (2007).

[36] A. F. Sadreev and A. S. Pilipchuk, Bound states in the continuum in zigzag quantum wire enforced by a finger gate, JETP Lett. 100, 585 (2015).

[37] W. Sheng and P. Hawrylak, Spin polarization in selfassembled quantum dots, Phys. Rev. B 73, 125331 (2006).

[38] S. Takahashi, R. S. Deacon, K. Yoshida, A. Oiwa, K. Shibata, K. Hirakawa, Y. Tokura, and S. Tarucha, Large Anisotropy of the Spin-Orbit Interaction in a Single InAs Self-Assembled Quantum Dot, Phys. Rev. Lett. 104, 246801 (2010).

[39] K. Gawarecki, Spin-orbit coupling and magnetic-field dependence of carrier states in a self-assembled quantum dot, Phys. Rev. B 97, 235408 (2018).

[40] P. Harvey-Collard, N. T. Jacobson, C. Bureau-Oxton, R. M. Jock, V. Srinivasa, A. M. Mounce, D. R. Ward, J. M. Anderson, R. P. Manginell, J. R. Wendt, T. Pluym, M. P. Lilly, D. R. Luhman, M. Pioro-Ladrière, and M. S. Caroll, Spin-Orbit Interactions for Singlet-Triplet Qubits in Silicon, Phys. Rev. Lett. 122, 217702 (2019).

[41] W. M. Witzel, I. Montaño, R. P. Muller, and M. S. Carroll, Multiqubit gates protected by adiabaticity and dynamical decoupling applicable to donor qubits in silicon, Phys. Rev. B 92, 081407(R) (2015).

[42] J. T. Muhonen, J. P. Dehollain, A. Laucht, S. Simmons, R. Kalra, F. E. Hudson, A. S. Dzurak, A. Morello, D. N. Jamieson, J. C. McCallum, and K. M. Itoh, Coherent control via weak measurements in ${ }^{31} \mathrm{P}$ single-atom electron and nuclear spin qubits, Phys. Rev. B 98, 155201 (2018). 
[43] B. Weber, Y.-L. Hsueh, T. F. Watson, R. Li, A. R. Hamilton, L. C. L. Hollenberg, R. Rahman, and M. Y. Simmons, Spin-orbit coupling in silicon for electrons bound to donors, npj Quant. Inf. 4, 61 (2018).

[44] K. W. Chan, W. Huang, C. H. Yang, J. C. C. Hwang, B. Hensen, T. Tanttu, F. E. Hudson, K. M. Itoh, A. Laucht, A. Morello, and A. S. Dzurak, Assessment of a Silicon Quantum Dot Spin Qubit Environment via Noise Spectroscopy, Phys. Rev. Appl. 10, 044017 (2018).

[45] P. Ross, B. C. Rose, C. C. Lo, M. L. W. Thewalt, A. M. Tyryshkin, S. A. Lyon, and J. L. Morton, Electron Spin Resonance of P Donors in Isotopically Purified Si Detected by Contactless Photoconductivity, Phys. Rev. Appl. 11, 054014 (2019).

[46] B. Hansen, W. W. Huang, C.-H. Yang, K. W. Chan, J. Yonedal, T. Tanttu, F. E. Hudson, A. Laucht, K. M. Itoh, T. D. Ladd, A. Morello, and A. S. Dzurak, A silicon quantum-dot-coupled nuclear spin qubit, Nat. Nanotech. 15, 13 (2020).

[47] B. C. Rose, A. M. Tyryshkin, H. Riemann, N. V. Abrosimov, P. Becker, H.-J. Pohl, M. L. W. Thewalt, K. M. Itoh, and S. A. Lyon, Coherent Rabi Dynamics of a Superradiant Spin Ensemble in a Microwave Cavity, Phys. Rev. X 7, 031002 (2017).

[48] D. V. Khomitsky, E. A. Lavrukhina, and E. Ya. Sherman, Electric dipole spin resonance at shallow donors in quantum wires, Phys. Rev. B 99, 014308 (2019).

[49] S. D. Ganichev and W. Prettl, Intense Terahertz Excitation of Semiconductors, Series on Semiconductor Science and Technology Vol. 14 (Oxford University Press, Oxford, UK, 2006).

[50] J. Takeda, K. Yoshioka, Y. Minami, and I. Katayama, Nanoscale electron manipulation in metals with intense THz electric fields, J. Phys. D: Appl. Phys. 51, 103001 (2018).

[51] A. Singh, A. Pashkin, S. Winnerl, M. Welsch, C. Beckh, P. Sulzer, A. Leitenstorfer, M. Helm, and H. Schneider, Up to $70 \mathrm{THz}$ bandwidth from an implanted Ge photoconductive antenna excited by a femtosecond Er:fibre laser, Light: Sci. Appl. 9, 30 (2020).

[52] M. Brooks and G. Burkard, Electric dipole spin resonance of two-dimensional semiconductor spin qubits, Phys. Rev. B 101, 035204 (2020).

[53] F. Mireles and G. Kirczenow, Ballistic spin-polarized transport and Rashba spin precession in semiconductor nanowires, Phys. Rev. B 64, 024426 (2001).

[54] M. M. Gelabert, L. Serra, D. Sánchez, and R. López, Multichannel effects in Rashba quantum wires, Phys. Rev. B 81, 165317 (2010).
[55] A. N. M. Zainuddin, S. Hong, L. Siddiqui, S. Srinivasan, and S. Datta, Voltage-controlled spin precession, Phys. Rev. B 84, 165306 (2011).

[56] L. Xu, Xin-Qi Li, and Qing-Feng Sun, Revisit the spinFET: Multiple reflection, inelastic scattering and lateral size effects, Sci. Rep. 4, 7527 (2014).

[57] I. Saïdi, S. B. Radhia, and K. Boujdaria, Band parameters of GaAs, InAs, InP, and InSb in the 40-band kp model, J. Appl. Phys. 107, 043701 (2010).

[58] M. A. Leontiadou, K. L. Litvinenko, A. L. Gilbertson, C. R. Pidgeon, W. R. Branford, L. F. Cohen, M. Fearn, T. Ashley, M. T. Emeny, B. N. Murdin, and S. K. Clowes, Experimental determination of the Rashba coefficient in $\mathrm{InSb} / \mathrm{InAlSb}$ quantum wells at zero magnetic field and elevated temperatures, J. Phys.: Condens. Matter 23, 035801 (2011).

[59] P. Wójcik, A. Bertoni, and G. Goldoni, Tuning Rashba spinorbit coupling in homogeneous semiconductor nanowires, Phys. Rev. B 97, 165401 (2018).

[60] L. D. Landau and E. M. Lifshitz, Quantum Mechanics Nonrelativistic Theory, 3rd ed., Course of Theoretical Physics Vol. 3 (Elsevier, Amsterdam, Netherlands, 1977).

[61] R. Winkler, Spin-Orbit Coupling Effects in Two-Dimensional Electron and Hole Systems (Springer, Berlin, 2003).

[62] For electrons, see: M. I. Dyakonov and V. Y. Kachorovskii, Spin relaxation of two-dimensional electrons on noncentrosymmetric semiconductors, Sov. Phys. Semicond. 20, 110 (1986).

[63] For holes: E. I. Rashba and E. Ya. Sherman, Spin-orbital band splitting in symmetric quantum wells, Phys. Lett. A 129, 175 (1988).

[64] N. B. Delone and V. P. Krainov, Atoms in Strong Light Fields, Springer Series in Chemical Physics (SpringerVerlag Berlin, Heidelberg, 1985).

[65] K. Blum, Density Matrix Theory and Applications, Springer Series on Atomic, Optical, and Plasma Physics (Springer, Berlin, 2012).

[66] E. N. Adams and E. I. Blount, Energy bands in the presence of an external force field II: Anomalous velocities, J. Phys. Chem. Solids 10, 286 (1959).

[67] S. A. Tarasenko, A. V. Poshakinskiy, E. L. Ivchenko, I. Stepanov, M. Ersfeld, M. Lepsa, and B. Beschoten, Zitterbewegung of spin split electrons, JETP Lett. 108, 326 (2018).

[68] M. Lewenstein, Ph. Balcou, M. Yu. Ivanov, A. L'Huillier, and P. B. Corkum, Theory of high-harmonic generation by low-frequency laser fields, Phys. Rev. A 49, 2117 (1994). 